\title{
Dynamic Matching and Bargaining Games: \\ A General Approach
}

Supplementary Online Appendix

Stephan Lauermann

April 15, 2012

This online Appendix contains technical material to supplement "Dynamic Matching and Bargaining Games: A General Approach." In particular, the Appendix provides a full characterization of the limit outcomes of the class of matching and bargaining games when signals contain information, that is, when $\eta<1$.

\section{Weak Convergence of Subsequences implies Weak Convergence of A SEQUENCE}

The following proposition restates Royden and Fitzpatrick (2010, Theorem 14, p. 171). I identify functions and their equivalence classes in this section.

PROPOSITION 3: Let $E$ be a measurable set and $1<p<\infty$. Then every bounded sequence in $L^{p}(E)$ has a subsequence that converges weakly in $L^{p}(E)$ to a function in $L^{p}(E)$.

The proof of the main characterization result uses the weak sequential compactness of $L^{2}[(0,1)]$ to conclude from weak subsequence convergence to convergence of the sequence. The technical argument is this.

CLAIM 4. Let $\left\{f_{k}\right\}$ be a sequence of measurable functions with elements in $\mathcal{F}=\{g$ : $[0,1] \rightarrow[0,1]\}$. The sequence $\left\{f_{k}\right\}$ converges weakly in $L^{2}([0,1])$ to a function $f$ in $L^{2}([0,1])$ if and only if every weakly convergent subsequence of $\left\{f_{k}\right\}$ converges to $f$.

PROOF: If $\left\{f_{k}\right\}$ converges weakly to $f$, so do all of its subsequences. Suppose that every weakly convergent subsequence of $\left\{f_{k}\right\}$ converges to $f$. I show that this implies that $\left\{f_{k}\right\}$ converges weakly to $f$. By contradiction: If $\left\{f_{k}\right\}$ does not converge weakly, then there is some function $g \in L^{2}([0,1])$ such that $\lim \int f_{k} g \neq \int f g$. By hypothesis, $f_{k}(x) \in[0,1]$, and, therefore, $\left\{\int f_{k} g\right\}$ is a sequence of numbers in $\left[-\int g, \int g\right]$. The Bolzano-Weierstrass theorem and $\lim _{k \rightarrow \infty} \int f_{k} g \neq \int f g$ imply the existence of a subsequence $\left\{f_{k^{\prime}}\right\}$ and a number $a \neq \int f g$ such that $\lim _{k^{\prime} \rightarrow \infty} \int f_{k^{\prime}} g=a$. Weak sequential compactness of $L^{2}([0,1])$ implies existence of a further subsequence $\left\{f_{k^{\prime \prime}}\right\}$ that converges weakly to some function $\hat{f}$. By hypothesis of the claim, $\hat{f}=f$. Hence, $\lim _{k^{\prime \prime} \rightarrow \infty} \int f_{k^{\prime \prime}} g=\int f g$ - a contradiction. 
Suppose matching is pairwise, $\zeta=0$, to simplify the exposition. The first feasibility condition is that ${ }^{31}$

$$
\int Q^{S}(c) d G^{S}(c)=\int Q^{B}(v) d G^{B}(v) .
$$

Suppose $\left(\Phi^{S}, \Phi^{B}\right)$ satisfy the steady-state conditions given strategy profile $\left(\sigma^{S}, \sigma^{B}\right)$. Let $A=\left(V^{S}, V^{B}, Q^{S}, Q^{B}\right)$ be the corresponding outcome. The trading probability satisfies

$$
Q^{B}(v)=q^{B}(v)+(1-\delta)\left(1-q^{B}(v)\right) q^{B}(v)+(1-\delta)^{2}\left(1-q^{B}(v)\right)^{2} q^{B}(v)+\ldots
$$

As in the proof of Lemma 4, the steady-state condition (1) implies that for every set $A \subset[0,1], \Phi^{B}(A) \leq \delta^{-1} G^{B}(A)$. Hence, the absolute continuity of $G^{B}$ implies absolute continuity of $\Phi^{B}$. Therefore, $\Phi^{B}$ admits a density $\phi^{B}$. Differentiating both sides of the steady-state condition implies that $\phi^{B}(v)=g^{B}(v)+(1-\delta)\left(1-q^{B}(v)\right) \phi^{B}(v)$ for almost all $v$.

Iterated application of the recursive equation implies

$$
\begin{aligned}
\phi^{B}(v) & =g^{B}(v)+(1-\delta)\left(1-q_{k}^{B}(v)\right) \phi^{B}(v) \\
& =g^{B}(v)+(1-\delta)\left(1-q_{k}^{B}(v)\right)\left(g^{B}(v)+(1-\delta)\left(1-q^{B}(v)\right)\right) \\
& =g^{B}(v)\left(1+(1-\delta)\left(1-q^{B}(v)\right)+(1-\delta)^{2}\left(1-q^{B}(v)\right)^{2}+\cdots\right),
\end{aligned}
$$

noting that $g^{B}(v)$ is bounded and $\lim _{n \rightarrow \infty}(1-\delta)^{n}\left(1-q^{B}(v)\right)^{n}=0$. Combining the previous observations,

$$
\begin{aligned}
& \int Q^{B}(v) d G^{B}(v) \\
= & \int\left(q^{B}(v)+(1-\delta)\left(1-q^{B}(v)\right) q^{B}(v)+(1-\delta)^{2}\left(1-q^{B}(v)\right)^{2} q^{B}(v)+\cdots\right) d G^{B}(v) \\
= & \int q^{B}(v)\left(1+(1-\delta)\left(1-q^{B}(v)\right)+(1-\delta)^{2}\left(1-q^{B}(v)\right)^{2}+\cdots\right) d G^{B}(v) \\
= & \int q^{B}(v) d \Phi^{B}(v) .
\end{aligned}
$$

Note that $\int Q^{B}(v) d G^{B}(v)=\int q^{B}(v) d \Phi^{B}(v)$ implies equality of the number of buyers who enter the market in any given period expecting to trade (the LHS) with the number of buyers who leave the market after having traded in any period (the RHS). An analogous argument proves $\int Q^{S}(c) d G^{S}(c)=\int q^{S}(c) d \Phi^{S}(c)$.

Let $\lambda(v, c)$ denote the probability of trade conditional on $v$ and $c$ being matched. By

${ }^{31}$ The numbering of equations continues the numbering in the main paper to facilitate cross-references. 
definition, $q^{S}(c)=\int \lambda(v, c) d \Phi^{B}(v) / M$. Rewriting (B1) using the previous implications of the steady-state conditions yields

$$
\begin{aligned}
\int Q^{S}(c) d G^{S}(c) & =\int Q^{B}(v) d G^{B}(v) \\
\Leftrightarrow \int q^{S}(c) d \Phi^{S}(c) & =\int q^{B}(v) d \Phi^{B}(v) \\
\Leftrightarrow \int\left(\int \lambda(v, c) d \Phi^{B}(v) / M\right) d \Phi^{S}(c) & =\int\left(\int(\lambda(v, c)) d \Phi^{S}(c) / M\right) d \Phi^{B}(v) .
\end{aligned}
$$

Therefore, the first feasibility condition (B1) holds. The second feasibility requirement is

$$
\int Q^{B}(v) P^{B}(v) d G^{B}(v)=\int Q^{S}(c) P^{S}(c) d G^{S}(c),
$$

noting that $V^{B}(v)=Q^{B}(v)\left(v-P^{B}(v)\right)$ and $V^{S}(c)=Q^{S}(c)\left(P^{S}(c)-c\right)$. Let $\rho(v, c)$ denote the expected price conditional on trade between $v$ and $c$ if $\lambda(v, c)>0$. If $\lambda(v, c)=0$, let $\rho(v, c)=0$. By definition, $P^{S}(c)=\int \rho(v, c) \frac{\lambda(v, c)}{q^{S}(c)} \frac{d \Phi^{B}(v)}{M}$. Using the steady state condition to rewrite (B2) analogously to before shows that

$\int\left(\int \rho(v, c) \lambda(v, c) d \Phi^{B}(v) / M\right) d \Phi^{S}(c)=\int\left(\int \rho(v, c) \lambda(v, c) d \Phi^{S}(c) / M\right) d \Phi^{B}(v)$,

which implies that the second feasibility condition (B2) holds as well.

\section{Omitted Steps of the Proof of Proposition 2}

\section{C1. Proof of Lemma 3 (Weak Incentive Compatibility) if $\eta<1$}

(i) Noisy Signals $(1 \geq \eta>0)$. - I consider the buyers' side. By construction of the signal, its c.d.f. is given by $F(\hat{v} \mid v, \eta)=G\left(\frac{\hat{v}-(1-\eta) v}{\eta}\right)$. Hence, its density is $f(\hat{v} \mid v, \eta)=$ $\frac{1}{\eta} g\left(\frac{\hat{v}-(1-\eta) v}{\eta}\right)$. Recall that $g(\varepsilon)=e^{-0.5|\varepsilon|}$. Take any pair $v^{\prime}$ and $v^{\prime \prime}$. The likelihood ratio of the signals is bounded,

$$
\frac{f\left(\hat{v} \mid v^{\prime}, \eta\right)}{f\left(\hat{v} \mid v^{\prime \prime}, \eta\right)}=\frac{g\left(\frac{\hat{v}-(1-\eta) v^{\prime}}{\eta}\right)}{g\left(\frac{\hat{v}-(1-\eta) v^{\prime}}{\eta}\right)}=e^{\frac{1}{2}\left|\frac{\hat{v}-(1-\eta) v^{\prime \prime}}{\eta}\right|-\frac{1}{2}\left|\frac{\hat{v}-(1-\eta) v^{\prime}}{\eta}\right|} \leq e^{\frac{1-\eta}{\eta}\left|v^{\prime}-v^{\prime \prime}\right|}
$$

using the (reverse) triangle inequality. Interchanging $v^{\prime}$ and $v^{\prime \prime}$ proves

$$
e^{-\frac{1-\eta}{\eta}\left|v^{\prime}-v^{\prime \prime}\right|} \leq \frac{f\left(\hat{v} \mid v^{\prime}, \eta\right)}{f\left(\hat{v} \mid v^{\prime \prime}, \eta\right)} \leq e^{\frac{1-\eta}{\eta}\left|v^{\prime}-v^{\prime \prime}\right|}
$$


Thus, signals are boundedly informative and the distribution of signals by similar types is similar.

Remark.-The bound (C2) implies that signals have a log-Lipschitz continuous density because $\left|\ln f\left(\hat{v} \mid v^{\prime}, \eta\right)-\ln f\left(\hat{v} \mid v^{\prime \prime}, \eta\right)\right| \leq \frac{1-\eta}{\eta}\left|v^{\prime}-v^{\prime \prime}\right|$. Since the subsequent arguments are based on (C2) only, the argument generalizes to all signals for which the signal densities are log-Lipschitz.

I show that trading probabilities $Q_{k}^{B}$ and prices $P_{k}^{B}$ are Lipschitz continuous in types. Recall $\sigma_{k}^{S}(c)=\left(p_{k}^{S}(\cdot, \cdot)(c), r_{k}^{S}(c)\right)$. When $\zeta=\beta=0$ (to simplify expressions), the per-period trading probability of type $v$ with action profile $\left(p^{B}, r^{B}\right)$ is

$$
q_{k}^{B}\left(v, p^{B}, r^{B}\right)=\iint 1_{p_{k}^{S}(\hat{v}, 1)(c) \leq r^{B}} f(\hat{v} \mid v, \eta) \frac{\phi_{k}^{S}(c)}{M_{k}} d \hat{v} d c .
$$

Take any pair $v^{\prime}$ and $v^{\prime \prime}$. An implication of (C2) is that the per-period trading probability satisfies

$$
e^{-\frac{1-\eta}{\eta} \mid v^{\prime}-v^{\prime \prime}} \mid q_{k}^{B}\left(v^{\prime}, p^{B}, r^{B}\right) \leq q_{k}^{B}\left(v^{\prime \prime}, p^{B}, r^{B}\right) \leq e^{\frac{1-\eta}{\eta}\left|v^{\prime}-v^{\prime \prime}\right|} q_{k}^{B}\left(v^{\prime}, p^{B}, r^{B}\right) .
$$

Rewriting $Q_{k}^{S}=q_{k}^{S}+\left(1-q_{k}^{S}\right)\left(1-\delta_{k}\right) Q_{k}^{S}$ shows

$$
Q_{k}^{B}\left(v^{\prime \prime}, p^{B}, r^{B}\right)=\frac{q_{k}^{B}\left(v^{\prime \prime}, p^{B}, r^{B}\right)}{\left(1-\delta_{k}\right) q_{k}^{B}\left(v^{\prime \prime}, p^{B}, r^{B}\right)+\delta_{k}} .
$$

Substituting from (C3) implies bounds on the lifetime trading probability,

$$
Q_{k}^{B}\left(v^{\prime \prime}, p^{B}, r^{B}\right) \geq \frac{e^{-\frac{1-\eta}{\eta}\left|v^{\prime}-v^{\prime \prime}\right|} q_{k}^{B}\left(v^{\prime}, p^{B}, r^{B}\right)}{\left(1-\delta_{k}\right) e^{-\frac{1-\eta}{\eta}\left|v^{\prime}-v^{\prime \prime}\right|} q_{k}^{B}\left(v^{\prime}, p^{B}, r^{B}\right)+\delta_{k}} \geq e^{-\frac{1-\eta}{\eta}\left|v^{\prime}-v^{\prime \prime}\right|} Q_{k}^{B}\left(v^{\prime}, p^{B}, r^{B}\right) .
$$

Analogously,

$$
Q_{k}^{B}\left(v^{\prime \prime}, p^{B}, r^{B}\right) \leq e^{\frac{1-\eta}{\eta}\left|v^{\prime}-v^{\prime \prime}\right|} Q_{k}^{B}\left(v^{\prime}, p^{B}, r^{B}\right) .
$$

When $\zeta=\beta=0$ (to simplify expressions), the expected trading price of type $v$ with bargaining strategy $\left(p^{B}, r^{B}\right)$ is

$$
P_{k}^{B}\left(v, p^{B}, r^{B}\right)=\iint 1_{p_{k}^{S}(\hat{v}, 1)(c) \leq r^{B}}\left[p_{k}^{S}(\hat{v}, 1)(c)\right] \frac{f(\hat{v} \mid v, \eta) \phi_{k}^{S}(c)}{q_{k}^{B}\left(v, p^{B}, r^{B}\right) M_{k}} d \hat{v} d c,
$$


whenever $q_{k}^{B}\left(v, p^{B}, r^{B}\right)>0$. For any pair $v^{\prime}$ and $v^{\prime \prime},(\mathrm{C} 2)$ and (C3) imply that

$$
\begin{aligned}
P_{k}^{B}\left(v^{\prime \prime}, p^{B}, r^{B}\right) & \leq \iint 1_{p_{k}^{S}(\hat{v}, 1)(c) \leq r^{B}}\left[p_{k}^{S}(\hat{v}, 1)(c)\right] \frac{e^{\frac{1-\eta}{\eta}\left|v^{\prime}-v^{\prime \prime}\right|} f\left(\hat{v} \mid v^{\prime}, \eta\right) \phi_{k}^{S}(c)}{e^{-\frac{1-\eta}{\eta}\left|v^{\prime}-v^{\prime \prime}\right|} q_{k}^{B}\left(v^{\prime}, p^{B}, r^{B}\right) M_{k}} d \hat{v} d c \\
& =e^{2 \frac{1-\eta}{\eta}\left|v^{\prime}-v^{\prime \prime}\right|} P_{k}^{B}\left(v^{\prime}, p^{B}, r^{B}\right) .
\end{aligned}
$$

The previous bounds on $Q_{k}^{B}$ and $P_{k}^{B}$ imply that for any action profile $\left(p^{B}, r^{B}\right)$,

$$
\begin{aligned}
& Q_{k}^{B}\left(v^{\prime \prime}, p^{B}, r^{B}\right)\left(v^{\prime \prime}-P_{k}^{B}\left(v^{\prime \prime}, p^{B}, r^{B}\right)\right) \\
\geq & e^{-\frac{1-\eta}{\eta} \mid v^{\prime}-v^{\prime \prime}} \mid Q_{k}^{B}\left(v^{\prime}, p^{B}, r^{B}\right) v^{\prime \prime}-e^{3 \frac{1-\eta}{\eta}\left|v^{\prime}-v^{\prime \prime}\right|} Q_{k}^{B}\left(v^{\prime}\right) P_{k}^{B}\left(v^{\prime}, p^{B}, r^{B}\right) .
\end{aligned}
$$

To simplify notation, let $P_{k}^{B}\left(v, p_{k}^{B}(v), r_{k}^{B}(v)\right)=P_{k}^{B}(v)$ and $Q_{k}^{B}\left(v, p_{k}^{B}(v), r_{k}^{B}(v)\right)=$ $Q_{k}^{B}(v)$. From this bound, the revealed preference condition (2) implies that

$$
\begin{aligned}
& V_{k}^{B}\left(v^{\prime \prime}\right)-V_{k}^{B}\left(v^{\prime}\right) \\
\geq & Q_{k}^{B}\left(v^{\prime \prime}, p_{k}^{B}\left(v^{\prime}\right), r_{k}^{B}\left(v^{\prime}\right)\right)\left(v^{\prime \prime}-P_{k}^{B}\left(v^{\prime \prime}, p_{k}^{B}\left(v^{\prime}\right), r_{k}^{B}\left(v^{\prime}\right)\right)\right)-Q_{k}^{B}\left(v^{\prime}\right)\left(v^{\prime}-P_{k}^{B}\left(v^{\prime}\right)\right) \\
\geq & Q_{k}^{B}\left(v^{\prime}\right)\left(e^{-\frac{1-\eta}{\eta}\left|v^{\prime}-v^{\prime \prime}\right|} v^{\prime \prime}-v^{\prime}\right)-Q_{k}^{B}\left(v^{\prime}\right) P_{k}^{B}\left(v^{\prime}\right)\left(e^{3 \frac{1-\eta}{\eta}\left|v^{\prime}-v^{\prime \prime}\right|}-1\right) \\
\geq & (-1)\left|v^{\prime}-v^{\prime \prime}\right| \frac{1+(1-\eta) 3 e^{3 \frac{1-\eta}{\eta}}}{\eta},
\end{aligned}
$$

where I used $e^{-\frac{1-\eta}{\eta} x} \geq 1-x \frac{1-\eta}{\eta}$ and $e^{\frac{1-\eta}{\eta} x}-1 \leq \frac{1-\eta}{\eta} e^{\frac{1-\eta}{\eta}} x$ when $\eta$ and $x$ are in $[0,1]$. Thus, payoffs are Lipschitz-continuous:

$$
0 \leq\left|V_{k}^{B}\left(v^{\prime}\right)-V_{k}^{B}\left(v^{\prime \prime}\right)\right| \leq \frac{1+(1-\eta) 3 e^{3 \frac{1-\eta}{\eta}}}{\eta}\left|v^{\prime}-v^{\prime \prime}\right| .
$$

Let $V_{k^{\prime}}^{B}$ be a pointwise convergent subsequence and suppose that $\lim Q_{k^{\prime}}^{B}\left(v^{\prime}, \sigma_{k^{\prime}}^{B}\right)=1$. Rearranging (C4) shows that

$$
\lim _{k^{\prime} \rightarrow \infty} Q_{k^{\prime}}^{B}\left(v^{\prime}, \sigma_{k^{\prime}}^{B}\right)=1 \Leftrightarrow \lim _{k^{\prime} \rightarrow \infty} \frac{\delta_{k^{\prime}}}{q_{k^{\prime}}^{B}\left(v^{\prime}, \sigma_{k^{\prime}}^{B}\right)}=0
$$

Thus, $\lim \frac{\delta_{k^{\prime}}}{q_{k^{\prime}}^{B}\left(v^{\prime}, \sigma_{k^{\prime}}^{B}\left(v^{\prime}\right)\right)}=0$. Equation (C3) implies that $\lim \frac{\delta_{k^{\prime}}}{q_{k^{\prime}}^{B}\left(v^{\prime \prime}, \sigma_{k^{\prime}}^{B}\left(v^{\prime}\right)\right)}=0$. Therefore, $\lim Q_{k^{\prime}}^{B}\left(v^{\prime \prime}, \sigma_{k^{\prime}}^{B}\left(v^{\prime}\right)\right)=1$. Furthermore, $\lim Q_{k^{\prime}}^{B}\left(v^{\prime \prime}, \sigma_{k^{\prime}}^{B}\left(v^{\prime}\right)\right)=1, \lim V_{k^{\prime}}^{B}\left(v^{\prime}\right)=\bar{V}^{B}\left(v^{\prime}\right)$, and $p_{k^{\prime}}^{B}\left(\hat{c}_{1}, \hat{c}_{2}\right)\left(v^{\prime}\right) \leq r_{k^{\prime}}^{B}\left(v^{\prime}\right)$ together imply that $\lim P_{k^{\prime}}^{B}\left(v^{\prime \prime}, \sigma_{k^{\prime}}^{B}\left(v^{\prime}\right)\right) \leq v^{\prime}-\bar{V}^{B}\left(v^{\prime}\right)$. 
Therefore, the revealed preference condition (2) implies

$$
\begin{aligned}
\bar{V}^{B}\left(v^{\prime \prime}\right) & \geq \liminf Q_{k^{\prime}}^{B}\left(v^{\prime \prime}, \sigma_{k^{\prime}}^{B}\left(v^{\prime}\right)\right)\left(v^{\prime \prime}-P_{k^{\prime}}^{B}\left(v^{\prime \prime}, \sigma_{k^{\prime}}^{B}\left(v^{\prime}\right)\right)\right) \\
& =\left(v^{\prime \prime}-\liminf P_{k^{\prime}}^{B}\left(v^{\prime \prime}, \sigma_{k^{\prime}}^{B}\left(v^{\prime}\right)\right)\right) \\
& \geq \bar{V}^{B}\left(v^{\prime}\right)+\left(v^{\prime \prime}-v^{\prime}\right) .
\end{aligned}
$$

Thus, the two sufficient conditions of Claim 3 hold, implying Weak Incentive Compatibility.

(II) Informative Signals AND Interior BARgaining Power $(1>\beta>0, \eta=0$, $\xi=0)$. The following arguments are standard; see, for example, Mortensen and Wright (2002). Buyers and sellers are symmetric and I concentrate on the buyer's side. Since $\eta=0$, I identify $\hat{v}=v$ and $\hat{c}=c$, and, since $\zeta=0$, I simplify $p_{k}^{B}(c, \varnothing)=p_{k}^{B}(c)$ and $p_{k}^{S}(v, 1)=p_{k}^{S}(v)$. The optimal offer strategy of a buyer satisfies $p_{k}^{B}(c)=r_{k}^{S}(c)$ if $v-r_{k}^{S}(c)>\left(1-\delta_{k}\right) V_{k}^{B}(v)$ and $p_{k}^{B}(c)<r_{k}^{S}(c)$ if $v-r_{k}^{S}(c)<\left(1-\delta_{k}\right) V_{k}^{B}(v)$. Offers in the remaining case $v-r_{k}^{S}(c)=\left(1-\delta_{k}\right) V_{k}^{B}(v)$ are payoff irrelevant for both the proposer and the responder. For a seller, $p_{k}^{S}(v)=r_{k}^{B}(v)$ if $r_{k}^{B}(v)-c>\left(1-\delta_{k}\right) V_{k}^{S}(c)$ and $p_{k}^{S}>r_{k}^{B}(v)$ if $r_{k}^{B}(v)-c<\left(1-\delta_{k}\right) V_{k}^{S}(c)$. Using the characterization of equilibrium reservation prices, payoffs satisfy

$$
\delta_{k} V_{k}^{B}(v)=\beta \int \max \left\{v-c-\left(1-\delta_{k}\right)\left(V_{k}^{S}(c)+V_{k}^{B}(v)\right), 0\right\} \frac{d \phi_{k}^{S}(c)}{M_{k}} .
$$

Fix some pair $v^{\prime}$ and $v^{\prime \prime}$. Let $D_{k}\left(v^{\prime}\right)=\left\{c \mid r_{k}^{S}(c) \leq p_{k}^{B}(c)\left(v^{\prime}\right)\right\}$. After rewriting,

$$
V_{k}^{B}\left(v^{\prime}\right)=\frac{\beta}{\delta_{k}+\beta\left(1-\delta_{k}\right) \frac{\Phi_{k}^{S}\left(D_{k}\left(v^{\prime}\right)\right)}{M_{k}}} \int_{D_{k}\left(v^{\prime}\right)}\left(v^{\prime}-p_{k}^{B}(c)\right) \frac{d \phi_{k}^{S}(c)}{M_{k}} .
$$

If a buyer with type $v^{\prime \prime}$ uses the same offer strategy as $v^{\prime}$ and if it accepts only prices of zero (which are offered with probability zero in equilibrium), then its payoffs are

$$
\frac{\beta}{\delta_{k}+\beta\left(1-\delta_{k}\right) \frac{\Phi_{k}^{S}\left(D_{k}\left(v^{\prime}\right)\right)}{M_{k}}} \int_{D_{k}\left(v^{\prime}\right)}\left(v^{\prime \prime}-p_{k}^{B}(c)\right) \frac{d \phi_{k}^{S}(c)}{M_{k}} .
$$

(C7) is a lower bound on equilibrium payoffs of $v^{\prime \prime}$. Combining the bound with (C6),

$$
V_{k}^{B}\left(v^{\prime \prime}\right) \geq V_{k}^{B}\left(v^{\prime}\right)+\frac{\beta \frac{\Phi_{k}^{S}\left(D_{k}\left(v^{\prime}\right)\right)}{M_{k}}}{\delta_{k}+\beta\left(1-\delta_{k}\right) \frac{\Phi_{k}^{S}\left(D_{k}\left(v^{\prime}\right)\right)}{M_{k}}}\left(v^{\prime \prime}-v^{\prime}\right) .
$$

Now Weak Incentive Compatibility follows from verifying the two conditions in Claim 3:

First, interchanging the role of $v^{\prime \prime}$ and $v^{\prime}$ in (C8) proves that each $V_{k}^{B}$ is Lipschitz 
continuous with a common Lipschitz constant one. Second, suppose that $\lim Q_{k}^{B}\left(v^{\prime}\right)=$ 1. From (C5) the per-period trading probability satisfies $\lim \frac{\delta_{k}}{q_{k}^{B}\left(v^{\prime}\right)}=\lim \frac{\delta_{k} M_{k}}{\Phi_{k}^{S}\left(D_{k}\left(v^{\prime}\right)\right)}=$ 0 . Thus, the coefficient on $\left(v^{\prime \prime}-v^{\prime}\right)$ on the right-hand side of (C8) converges to one, proving that $\bar{V}^{B}\left(v^{\prime \prime}\right) \geq \bar{V}_{k}^{B}\left(v^{\prime}\right)+\left(v^{\prime \prime}-v^{\prime}\right)$ whenever $V_{k^{\prime}}^{B}\left(v^{\prime \prime}\right)$ and $V_{k^{\prime}}^{B}\left(v^{\prime}\right)$ converge pointwise.

(III) Informative Signals AND COMPETITION $(1>\beta \geq 0, \eta=0, \xi>0)$.

Exposition is simplified by considering $\beta=0$ first, so that sellers are always proposers. If a seller of type $c^{\prime \prime}$ chooses the same offer strategy as a type $c^{\prime}$, the seller trades at the same price and with the same probability. Thus, optimality of equilibrium actions implies

$$
V_{k}^{S}\left(c^{\prime \prime}\right) \geq V_{k}^{S}\left(c^{\prime}\right)+Q_{k}^{S}\left(c^{\prime}\right)\left(c^{\prime}-c^{\prime \prime}\right) .
$$

Interchanging $c^{\prime}$ and $c^{\prime \prime}$, it follows that $V_{k}^{S}$ is Lipschitz continuous with constant one. In addition, $\lim Q_{k}^{S}\left(c^{\prime}\right)=1$ implies $\bar{V}^{S}\left(c^{\prime \prime}\right) \geq \bar{V}^{S}\left(c^{\prime}\right)+\left(c^{\prime}-c^{\prime \prime}\right)$ if payoffs converge pointwise. Thus, Weak Incentive Compatibility holds. The argument for this case is identical to the argument when $\eta=1$ (signals are uninformative).

The following observations are helpful when considering the buyers' side. From (C9), $V_{k}^{S}$ is decreasing with a slope bounded between minus one and zero. The equilibrium requirement $r_{k}^{S}(c)=c+\left(1-\delta_{k}\right) V_{k}^{S}(c)$ implies that the reservation price is continuous and strictly increasing, with a slope between $\delta_{k}$ and one. Therefore, $r_{k}^{S}(c)-r_{k}^{S}\left(c^{\prime}\right) \leq c-c^{\prime}$. In particular, $r_{k}^{S}$ has a continuous inverse $\left(r_{k}^{S}\right)^{-1}$ on $\left[r_{k}^{S}(0), 1\right]$. The inverse is extended to all of $[0,1]$ by setting $\left(r_{k}^{S}\right)^{-1}(r)=0$ for all $r \leq r_{k}^{S}(0)$.

Consider the buyers' side. If a buyer is matched with a single seller, the equilibrium price offer is given by $p_{k}^{S}(v)=r_{k}^{B}(v)$ if $r_{k}^{B}(v)-c>\left(1-\delta_{k}\right) V_{k}^{S}(c)$ and $p_{k}^{S}>r_{k}^{B}(v)$ if $r_{k}^{B}(v)-c<\left(1-\delta_{k}\right) V_{k}^{S}(c)$. If the buyer is matched with two sellers, the resulting interaction is equivalent to a reverse first-price auction with two bidders and a reservation price $r_{k}^{B}(v)$ (the buyer rejects all offers which are higher). The opportunity cost of the sellers (bidders) are $c+\left(1-\delta_{k}\right) V_{k}^{S}(c)=r_{k}^{S}(c)$. Let $r_{(1)}$ denote the minimum of the two sellers' opportunity costs and let $r_{(2)}$ be the maximum. Define $\zeta_{k}=\zeta \frac{\Phi_{k}^{S}(1)}{M_{k}}$. The distribution of $r_{(1)}$ is

$$
F_{k}(r)=\left(\frac{\left(1-\Phi\left(\left(r_{k}^{S}\right)^{-1}(r)\right)\right)}{\Phi_{k}^{S}(1)}\right)^{2} .
$$

Standard characterization of equilibrium in first-price auctions (using the payoff equivalence to second-price auctions) implies that the price offer (the bidding strategy) is $p_{k}^{S}(v, 2)(c)=E_{k}\left[\min \left\{c^{\prime}, r_{k}^{B}(v)\right\} \mid c^{\prime}>c\right]$ if $r_{k}^{B}(v)>r_{k}^{S}(c) .{ }^{32}$ If $r_{k}^{B}(v)<r_{k}^{S}(c)$, any price offer above $r_{k}^{B}(v)$ is optimal. In equilibrium, a buyer trades whenever $r_{(1)} \leq r_{k}^{B}(v)$. The expected price conditional on trading is $P_{k}^{B}=E_{k}\left[\min \left\{r_{(2)}, r_{k}^{B}\right\} \mid r_{(1)} \leq r_{k}^{B}\right]$. Thus,

\footnotetext{
${ }^{32}$ See, for example, Vijay Krishna (2010, p. 21 "Reserve Prices"), Auction Theory, 2nd Edition, Academic Press.
} 
a buyer's payoff satisfies

$$
V_{k}^{B}(v)=\zeta_{k} F_{k}(r)\left(v-E_{k}\left[\min \left\{r_{(2)}, r\right\} \mid r_{(1)} \leq r\right]\right)+\left(1-\zeta_{k} F_{k}(r)\right)\left(1-\delta_{k}\right) V_{k}^{B}(v),
$$

at $r=r_{k}^{B}(v)$. Rewriting and substituting the equilibrium condition for $r=r_{k}^{B}(v)$,

$$
\delta_{k} V_{k}^{B}(v)=\zeta_{k} F_{k}(r)\left(r-E_{k}\left[\min \left\{r_{(2)}, r\right\} \mid r_{(1)} \leq r\right]\right) .
$$

The derivative of RHS(C10) with respect to $r$ can be shown to be $\zeta_{k}\left(\Phi_{k}^{S}\left(\left(r_{k}^{S}\right)^{-1}(r)\right) / \Phi_{k}^{S}(1)\right)^{2} \geq$

0 . In particular, the RHS is weakly increasing in $r$. The characterization (C10) implies the two conditions of Claim 3.

First, $V_{k}^{B}$ is Lipschitz continuous with constant $1 /\left(1-\delta_{k}\right)$. To see why, note that $V_{k}^{B}$ must be weakly increasing. Suppose not. If $V_{k}^{B}$ is strictly decreasing instead, $r_{k}^{B}(v)$ is strictly increasing, recall that $r_{k}^{B}(v)=v-\left(1-\delta_{k}\right) V_{k}^{B}$. Therefore, RHS(C10) is weakly increasing in $v$ after substituting $r=r_{k}^{B}(v)$, while the LHS(C10) is strictly decreasinga contradiction. Second, $V_{k}^{B}$ is increasing with a slope less than $1 /\left(1-\delta_{k}\right)$. Suppose not. Then, $r_{k}^{B}(v)$ would be strictly decreasing, and therefore RHS(C10) would be weakly decreasing; a contradiction. Thus, the first condition of Claim 3 holds.

Second, suppose that $V_{k}^{S}$ and $V_{k}^{B}$ converge pointwise. For convenience, suppose that the pointwise limit is equal to $\bar{V}^{S}$ and $\bar{V}^{B}$ everywhere. Take any pair of types $v^{\prime}$ and $v^{\prime \prime}$ and suppose that $\lim Q_{k}^{B}\left(v^{\prime}\right)=1$. If $v^{\prime \prime}<v^{\prime}$, then $V_{k}^{B}\left(v^{\prime \prime}\right) \geq V_{k}^{B}\left(v^{\prime}\right)+\frac{1}{1-\delta_{k}}\left(v^{\prime \prime}-v^{\prime}\right)$ follows from the fact that $V_{k}^{B}$ is increasing with a slope less than one. Taking limits implies $\bar{V}^{B}\left(v^{\prime \prime}\right) \geq \bar{V}^{B}\left(v^{\prime}\right)+\left(v^{\prime \prime}-v^{\prime}\right)$, as required in Claim 3. So, suppose that $v^{\prime \prime}>v^{\prime}$. Pointwise convergence of payoffs implies that $r_{k}^{S}$ and $r_{k}^{B}$ converge pointwise as well. Let $r_{L}=\lim r_{k}^{B}\left(v^{\prime}\right)$ and $r_{H}=\lim r_{k}^{B}\left(v^{\prime \prime}\right)$. I show that $r_{L}=r_{H}$, which implies $\bar{V}^{B}\left(v^{\prime \prime}\right)=$ $\bar{V}^{B}\left(v^{\prime}\right)+\left(v^{\prime \prime}-v^{\prime}\right)$. By contradiction. If $r_{L} \neq r_{H}$, then $r_{L}<r_{H}$, from the fact that $V_{k}^{B}$ is increasing with a slope less than one. Choose $r_{l}$ and $r_{h}$ such that $r_{L}<r_{l}<r_{h}<r_{H}$. Let $c_{L}=\lim \left(r_{k}^{S}\right)^{-1}\left(r_{k}^{B}\left(v^{\prime}\right)\right)$. Define $c_{l}=\lim \left(r_{k}^{S}\right)^{-1}\left(r_{l}\right)$ and define $c_{h}$, and $c_{H}$ analogously. $\lim Q_{k}^{B}\left(v^{\prime}\right)=1$ implies $Q_{k}^{B}\left(v^{\prime}\right)>0$ sufficiently deep into the sequence, and, hence, $r_{k}^{S}(0)<r_{k}^{B}\left(v^{\prime}\right)$ for all $k$ sufficiently large. From the previous observation that $r_{k}^{S}$ is increasing with a slope less than one on $\left[r_{k}^{S}(0), 1\right], c_{h}-c_{l} \geq r_{h}-r_{l}>0$. Sellers' payoffs satisfy Weak Incentive Compatibility. Thus $r_{L}=\bar{V}^{S}\left(c_{L}\right)+c_{L}<\bar{V}^{S}\left(c_{l}\right)+c_{l}$ requires $\bar{Q}^{S}(c)<1$ for all $c \in\left(c_{l}, c_{h}\right)$. Therefore, Claim $1, c_{l}<c_{h}$, and $G^{S}\left(c_{h}\right)-G^{S}\left(c_{l}\right)>0$ together imply $\liminf \frac{\Phi_{k}^{S}(c)}{M_{k}}>0$ for all $c \in\left(c_{l}, c_{h}\right)$. Moreover, sufficiently deep into the sequence, $r_{k}^{S}(c) \leq r_{h}<r_{H}$ for all $c<c_{h}$. Therefore, $\liminf \frac{\Phi_{k}^{S}(c)}{M_{k}}>0$ for all $c \in\left(c_{l}, c_{h}\right)$ implies that $\lim \sup \left(r_{H}-E_{k}\left[\min \left\{r_{(2)}, r_{k}^{B}\left(v^{\prime \prime}\right)\right\} \mid r_{(1)} \leq r_{k}^{B}\left(v^{\prime \prime}\right)\right]\right) \geq\left(r_{H}-r_{h}\right)>0$. It also implies that $\liminf \zeta_{k} F_{k}\left(r_{k}^{B}\left(v^{\prime \prime}\right)\right)>0$. Thus, if $r_{H} \neq r_{L}$ then the RHS(C10) would stay positive at $v^{\prime \prime}$ in the limit, whereas the LHS converges to zero-a contradiction. Thus, the second condition of Claim 3 holds.

Finally, consider the case where $1>\beta>0$, in addition to $\eta=0$ and $\xi>0$. Fix some 
type $c$. Let $B_{k}=\left\{v \mid r_{k}^{B}(v) \geq r_{k}^{S}(c)\right\}$. The payoff of a seller having type $c$ satisfies

$\delta V_{k}^{S}(c)=(1-\beta) \int_{v \in B_{k}}\left((1-\zeta)\left(r_{k}^{B}(v)-r_{k}^{S}(c)\right)+\zeta\left(p_{k}^{S}(v, 2)(c)-r_{k}^{S}(c)\right) \frac{1-\Phi_{k}^{S}(c)}{\Phi_{k}^{S}(1)}\right) \frac{\phi_{k}^{B}(v)}{M_{k}} d v$.

Weak Incentive Compatibility follows for sellers analogously to the reasoning for Weak Incentive Compatibility in Case (ii) for the buyers' side when $\zeta=0$ and $\beta \in(0,1)$.

Consider the buyers' side and fix some type $v$. Let $D_{k}=\left\{c \mid r_{k}^{B}(v) \geq r_{k}^{S}(c)\right\}$. The payoff of a type $v$ satisfies

$$
\begin{aligned}
\delta_{k} V_{k}^{B}(v)= & (1-\beta) \zeta_{k} F_{k}(r)\left(r-E_{k}\left[\min \left\{r_{(2)}, r\right\} \mid r_{(1)} \leq r\right]\right) \\
& +\beta \int_{c \in D_{k}}\left(r-r_{k}^{S}(c)\right) \frac{\phi_{k}^{S}(c)}{M_{k}} d c,
\end{aligned}
$$

at $r=r_{k}^{B}(v)$. Weak Incentive Compatibility follows analogously to before when $\beta=0$. In particular, the RHS of (C11) is increasing in $r$, which implies Lipschitz continuity of $V_{k}^{B}$ with Lipschitz constant one.

\section{C2. Proof of Lemma 5 (Non-Competitive Outcomes)}

CASE $1(\eta=\beta=\zeta=0)$. The optimal price offer of a seller satisfies $p_{k}^{S}(v, 1) \geq$ $r_{k}^{B}(v)$. Hence, $P_{k}^{B}\left(v, \sigma_{k}^{B}(v)\right) \geq r_{k}^{B}(v)$. The payoff of buyers is therefore bounded above,

$$
V_{k}^{B}(v) \leq Q_{k}^{B}(v)\left(v-r_{k}^{B}(v)\right) \leq\left(v-\left(v-(1-\delta) V_{k}^{B}(v)\right)\right)=\left(1-\delta_{k}\right) V_{k}^{B}(v) .
$$

Thus, $V_{k}^{B}(v)=0$ for all $v$ and for all $k$. Hence, $\bar{V}^{B}(v)=0$ for almost all $v$, and, in particular, $\bar{V}^{B}$ is not equivalent to the competitive payoff, $\max \left\{v-p^{w}, 0\right\}$.

CASE $2(\eta=1-\beta=0)$. The optimal price offer of a buyer satisfies $p_{k}^{B}\left(c_{1}, c_{2}\right) \leq$ $\min \left\{r_{k}^{S}\left(c_{1}\right), r_{k}^{S}\left(c_{2}\right)\right\}$ and $p_{k}^{B}\left(c_{1}, \emptyset\right) \leq \min r_{k}^{S}\left(c_{1}\right)$. Hence, $P_{k}^{S}\left(c, \sigma_{k}^{S}(c)\right) \leq r_{k}^{S}(c)$. The payoff of sellers is therefore bounded above,

$$
V_{k}^{S}(c) \leq Q_{k}^{S}(c)\left(r_{k}^{S}(c)-c\right) \leq\left(1-\delta_{k}\right) V_{k}^{S}(c) .
$$

Thus, $V_{k}^{S}(c)=0$ for all $c$ and for all $k$. Hence, $\bar{V}^{S}(c)=0$ for almost all $c$, and, in particular, $\bar{V}^{S}$ is not equivalent to the competitive payoff, $\max \left\{p^{w}-c, 0\right\}$.

\section{STATEMENT AND PROOF OF CHARACTERIZATION WITH ENTRY}

Section IV.B sketches a modification of the characterization result in the context of models with entry. I provide the formal details here.

Weak Pairwise Efficiency with Entry.-For every weak limit $\bar{A}: \bar{V}^{S}(c)+\bar{V}^{B}(v) \geq v-c$ for almost all pairs $(c, v)$ for which either $\bar{Q}^{S}(c) \in(0,1)$ or $\bar{Q}^{B}(v) \in(0,1)$ (or both). 
Bayesian Incentive Compatibility.-For every weak limit $\bar{A}$ : (i) $\bar{Q}^{S}$ is equivalent to a non-increasing function and $\bar{Q}^{B}$ is equivalent to a non-decreasing function and (ii) for almost all $c$ and $v,{ }^{33}$

$$
\begin{aligned}
& \bar{V}^{S}(c)=\bar{V}^{S}(1)+\int_{c}^{1} \bar{Q}^{S}(\tau) d \tau, \\
& \bar{V}^{B}(v)=\bar{V}^{B}(0)+\int_{0}^{v} \bar{Q}^{B}(\tau) d \tau .
\end{aligned}
$$

Payoffs without Trade are Zero.-For almost all $c, Q_{k}^{S}(c)=0$ implies $V_{k}^{S}(c)=0$ and for almost all $v, Q_{k}^{B}(v)=0$ implies $V_{k}^{B}(v)=0$.

Non-vanishing Trading Volume.-The sequence satisfies ${ }^{34}$

$$
\lim _{k \rightarrow \infty} \inf \int Q_{k}^{S}(c) d G^{S}+\int Q_{k}^{B}(v) d G^{B}>0 .
$$

PROPOSITION 4: Suppose $\left\{A_{k}\right\}$ is a sequence of feasible outcomes, the trading volume is non-vanishing, and payoffs without trade are zero. Then the sequence converges weakly to the competitive outcome if and only if the sequence satisfies Bayesian Incentive Compatibility and Weak Pairwise Efficiency with Entry.

ProOF Let $\bar{A}$ be the limit of some weakly convergent subsequence as in the proof of Proposition 1. Let $c_{*}$ and $v_{*}$ be defined as before,

$$
\begin{aligned}
& c_{*}=\sup \left\{c \in[0,1] \mid \exists \varepsilon>0 \text { s.t. } \bar{Q}^{S}\left(c^{\prime}\right)=1 \text { for almost all } c^{\prime} \in B_{\varepsilon}(c)\right\}, \\
& v_{*}=\inf \left\{v \in[0,1] \mid \exists \varepsilon>0 \text { s.t. } \bar{Q}^{B}\left(v^{\prime}\right)=1 \text { for almost all } v^{\prime} \in B_{\varepsilon}(v)\right\},
\end{aligned}
$$

with $\sup \emptyset=0$ and $\inf \emptyset=1$. Let $c_{0}$ and $v_{0}$ be defined as

$$
\begin{aligned}
& c_{0}=\inf \left\{c \in[0,1] \mid \exists \varepsilon>0 \text { s.t. } \bar{Q}^{S}\left(c^{\prime}\right)=0 \text { for almost all } c^{\prime} \in B_{\varepsilon}(c)\right\}, \\
& v_{0}=\sup \left\{v \in[0,1] \mid \exists \varepsilon>0 \text { s.t. } \bar{Q}^{B}\left(v^{\prime}\right)=0 \text { for almost all } v^{\prime} \in B_{\varepsilon}(v)\right\} .
\end{aligned}
$$

The monotonicity requirement of Bayesian Incentive Compatibility implies that it is without further loss of generality to assume that $\bar{Q}^{S}(c)=1$ for all $c<c_{*}, \bar{Q}^{S}(c) \in$ $(0,1)$ for all $c \in\left(c_{*}, c_{0}\right)$, and $\bar{Q}^{S}(c)=0$ for all $c>c_{0}$. Similarly, $\bar{Q}^{B}(v)=1$ for all $v \geq v_{*}, \bar{Q}^{B}(v) \in(0,1)$ for all $v \in\left(v_{0}, v_{*}\right)$, and $\bar{Q}^{B}(v)=0$ for all $v<v_{0}$. By (D1) and (D2), one can further assume that $\bar{V}^{S}$ and $\bar{V}^{B}$ are continuous.

I show that limit outcomes are pairwise efficient in the following.

\footnotetext{
${ }^{33}$ The conditions (D1) and (D2) are stronger than needed. The argument requires only that the following conditions are true almost everywhere: $a-b \leq \bar{V}^{S}(b)-\bar{V}^{S}(a) \leq 0$ if $a \leq b$ and $\bar{V}^{S}\left(c^{\prime}\right) \geq \bar{V}^{S}(c)+\left(c-c^{\prime}\right)$ for pairs $\left(c, c^{\prime}\right)$ for which $\bar{Q}^{S}(c)=1$. Similarly, $0 \leq \bar{V}^{B}(b)-\bar{V}^{B}(a) \leq b-a$ if $a \leq b$ and $\bar{V}^{B}\left(v^{\prime}\right) \geq \bar{V}^{B}(v)+\left(v^{\prime}-v\right)$ for pairs $\left(v^{\prime}, v\right)$ for which $\bar{Q}^{B}(v)=1$.

${ }^{34}$ If outcomes are feasible, this is equivalent to $\lim _{k \rightarrow \infty} \inf \int Q_{k}^{S}(c) d G^{S}>0$.
} 
Case 1. Suppose either $v_{*}>v_{0}$ or $c_{*}<c_{0}$, or both. Suppose that $v_{*}>v_{0}$ for concreteness. Then, for any $\varepsilon$ small enough, Weak Pairwise Efficiency with Entry implies $\bar{V}^{S}(0)+\bar{V}^{B}\left(v_{*}-\varepsilon\right) \geq v_{*}-\varepsilon-0$. Thus, continuity implies $\bar{V}^{S}(0)+\bar{V}^{B}\left(v_{*}\right) \geq v_{*}$. (D2) implies further that

$$
\bar{V}^{S}(0)+\bar{V}^{B}(1) \geq 1 .
$$

(D1) and (D2) imply that the absolute value of the slope of $\bar{V}^{S}$ and $\bar{V}^{B}$ are weakly smaller than one. Therefore,

$$
\begin{aligned}
\bar{V}^{S}(c) & \geq \bar{V}^{S}(0)-c, \\
\bar{V}^{B}(v) & \geq \bar{V}^{B}(1)-(1-v) .
\end{aligned}
$$

(D3), (D4), and (D5) imply

$$
\bar{V}^{B}(v)+\bar{V}^{S}(c) \geq \bar{V}^{S}(0)-c+\bar{V}^{B}(1)-(1-v) \geq v-c .
$$

Case 2a. Suppose that $v_{*}=v_{0}, c_{*}=c_{0}$ and $v_{*} \leq c_{*}$. (D1) and (D2) imply that $\bar{V}^{B}(v) \geq v-v_{*}$ for all $v$ and $V^{S}(c) \geq v_{*}-c$ for all $c$. Hence,

$$
\bar{V}^{B}(v)+\bar{V}^{S}(c) \geq v-v_{*}+v_{*}-c=v-c .
$$

Case 2b. Suppose that $v_{*}=v_{0}, c_{*}=c_{0}$ and $v_{*}>c_{*}$. This case is shown to imply a contradiction, that is, this case does not arise in equilibrium. Since payoffs without trade are zero and payoffs are continuous, $\bar{V}^{S}(c)=0$ for all $c \geq c_{*}$ and $\bar{V}^{B}(v)=0$ for all $v \leq$ $v_{*}$. This, (D1) and (D2), imply that $\bar{V}^{S}(c)=c_{*}-c$ for all $c \leq c_{*}$ and $\bar{V}^{B}(v)=v-v_{*}$ for all $v \geq v_{*}$. By hypothesis, $\bar{Q}^{S}(c)=1$ if $c<c_{*}$ and $\bar{Q}^{S}(c)=0$ if $c>c_{*}$. Similarly, $\bar{Q}^{B}(v)=1$ if $v>v_{*}$ and $\bar{Q}^{B}(v)=0$ if $v<v_{*}$. Therefore, $\int \bar{Q}^{S}(c) d G^{S}(c)=$ $G^{S}\left(c_{*}\right)$ and $\int \bar{Q}^{B}(v) d G^{B}(v)=G^{B}(1)-G^{B}\left(v_{*}\right)$. Feasibility extends to the weak limit. The feasibility condition $\int \bar{Q}^{S}(c) d G^{S}(c)=\int \bar{Q}^{B}(v) d G^{B}(v)$ implies $G^{S}\left(c_{*}\right)=$ $G^{B}(1)-G^{B}\left(v_{*}\right)$. The assumption that the trading volume is non-vanishing implies that $G^{S}\left(c_{*}\right)>0$. The previous observations imply 


$$
\begin{aligned}
& \int_{v_{*}}^{1} \bar{V}^{B}(v) d G^{B}(v)+\int_{0}^{c_{*}} \bar{V}^{S}(c) d G^{S}(c) \\
= & \int_{v_{*}}^{1}\left(v-v_{*}\right) d G^{B}(v)+\int_{0}^{c_{*}}\left(c_{*}-c\right) d G^{S}(c) \\
= & \int_{v_{*}}^{1} v d G^{B}(v)-\int_{0}^{c_{*}} c d G^{S}(c)+\left[\int_{0}^{c_{*}} c_{*} d G^{S}(c)-\int_{v_{*}}^{1} v_{*} d G^{B}(v)\right] \\
= & \int_{v_{*}}^{1} v d G^{B}(v)-\int_{0}^{c_{*}} c d G^{S}(c)+\left[c_{*} G^{S}\left(c_{*}\right)-v_{*}\left(1-G^{B}\left(v_{*}\right)\right)\right] \\
(\mathrm{D} 6)< & \int_{v_{*}}^{1} v \bar{Q}^{B} d G^{B}(v)-\int_{0}^{c_{*}} c \bar{Q}^{S} d G^{S}(c)
\end{aligned}
$$

The first equality follows from the observations about the payoffs of inframarginal types in the paragraph before. The strict inequality follows from the hypothesis that $v_{*}>c_{*}$, from the feasibility implication $G^{S}\left(c_{*}\right)=\left(1-G^{B}\left(v_{*}\right)\right)$, and from $G^{S}\left(c_{*}\right)>0$.

The second requirement of feasibility is that $\int\left(v \bar{Q}^{B}(v)-\bar{V}^{B}(v)\right) d G^{B}(v)$ must be equal to $\int\left(\bar{V}^{S}(c)+c \bar{Q}^{S}(c)\right) d G^{S}(c)$, which requires that

$$
\int \bar{V}^{S}(c) d G^{S}(c)+\int \bar{V}^{B}(v) d G^{B}(v)=\int v \bar{Q}^{B}(v) d G^{B}(v)-\int c \bar{Q}^{S}(c) d G^{S}(c) .
$$

(D6) is in contradiction with (D7). Thus, $v_{*}>c_{*}$ cannot hold in equilibrium.

The previous observations imply that the limit outcome is pairwise efficient in all cases that can arise in equilibrium. By Lemma 1, the limit of any weakly convergent subsequence is competitive. As in the proof of Proposition 1, this implies that the sequence of outcomes converges weakly to the competitive outcome. Again, necessity of the conditions is immediate. 